
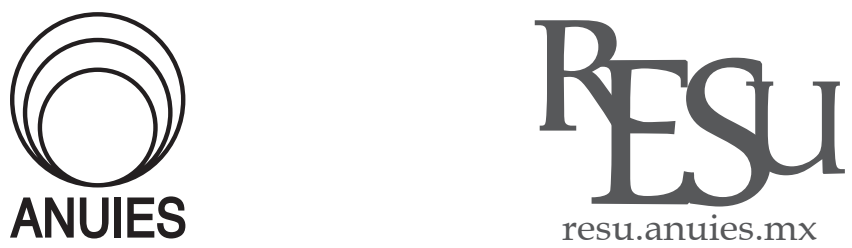

ARTí́CULO

\title{
Saberes-haceres de egresados de la Universidad Veracruzana Intercultural en posgrados no interculturales: tensiones y sinergias
}

\author{
Knowledge-activities of graduates of the Universidad Veracruzana \\ Intercultural in non intercultural postgraduate degrees: tensions and \\ synergies \\ Rosa Guadalupe Mendoza Zuany, Gunther Dietz, Laura Selene Mateos Cortés \\ Universidad Veracruzana, Xalapa, México \\ Correos electrónicos: guntherdietz@gmail.com, lupitamendoza.zuany@gmail.com, lauramat@gmail.com
}

Recibido el 04 de diciembre del 2018; aceptado el 25 de septiembre del 2019

\begin{abstract}
Resumen
El objetivo de este artículo es describir y analizar las tensiones que han experimentado egresados de la Universidad Veracruzana Intercultural (UVI) que cursan posgrados no interculturales, así como las sinergias que han contribuido a que complementen su formación de licenciatura. Esto ocurre al desarrollar saberes-haceres específicos construidos en un programa con enfoque intercultural que entran en contacto con saberes académicos. Se entrevistó de forma semi-estructurada a egresados de la uvi que han continuado sus estudios de posgrado. Se presenta un balance de los egresados sobre sus saberes-haceres, así como las tensiones y las sinergias que se producen durante sus estudios de posgrado cuando entran en contacto con saberes académicos.
\end{abstract}

Palabras clave: Saberes-haceres; Saberes académicos; Universidades interculturales; Posgrados no interculturales; Estudiantes indígenas 


\begin{abstract}
This article objective of is to describe and analyze the tensions experienced by graduates of the Universidad Veracruzana Intercultural (UVI) who have studied non-intercultural postgraduate degrees, as well as the synergies that contribute to complement their undergraduate education. This happens when developing specific knowledge-activities constructed in a program with an intercultural approach that come into contact with academic knowledge. Semi-structured interviews were given to graduates of the UVI who have continued their postgraduate studies. A balance of the graduates is presented about their knowledge-activities, the tensions and the synergies that occur in their postgraduate studies when they come into contact with academic knowledge.
\end{abstract}

Keywords: Knowledge-activities; Academic knowledge; Intercultural universities; Non-intercultural postgraduate studies; Indigenous students

\title{
Introducción
}

$\mathrm{E}$ l objetivo de este artículo es describir y analizar las tensiones que han experimentado egresados de la Universidad Veracruzana Intercultural (UVI) - en la Licenciatura en Gestión Intercultural para el Desarrollo (LGID) - que han cursado posgrados no interculturales, así como las sinergias que han contribuido a que complementen su formación de licenciatura. Esto ocurre al portar saberes-haceres específicos construidos y/o fortalecidos en un programa de licenciatura con enfoque intercultural que entran en contacto con saberes académicos de posgrados que no se consideran interculturales.

Las universidades interculturales son producto de una política que se formula desde la Secretaría de Educación Pública de México, a través de la Coordinación General de Educación Intercultural y Bilingüe (CGEIB), a partir de 2002, para atender la demanda de la población indígena de una educación pertinente a nivel superior y que se asocia a otras demandas que emergieron a partir de 1994 con el levantamiento zapatista. Este tipo de instituciones educativas, junto a otras respuestas institucionales desde las universidades convencionales para la atención de demandas educativas de los pueblos indígenas, se han configurado en varios países de América Latina (Mato, 2009).

La UVI es una entidad académica de la Universidad Veracruzana (Uv), a diferencia de otras universidades interculturales en México que no forman parte de la estructura de una universidad pública, que ha ofrecido la Licenciatura en Gestión Intercultural para el Desarrollo (LGID) en sus cuatro sedes regionales en el estado de Veracruz (Grandes Montañas, Las Selvas, Totona- 
capan y Huasteca) con cinco especialidades como opciones terminales: comunicación, derechos, lenguas, salud y sustentabilidad. Fundada en 2005, uno de sus rasgos distintivos es su oferta educativa orientada a formar nuevos perfiles requeridos en las zonas indígenas para la gestión y la mediación de una transformación social definida por los actores regionales de formas diversas, y en ocasiones contradictorias, y a enfatizar el fortalecimiento de las lenguas indígenas y de sus prácticas culturales y locales. Se entrelazan procesos de investigación estudiantil, docencia que se orienta por la idea de un aprendizaje situado y vinculación comunitaria para lograr procesos pertinentes deliberados por los actores locales. Varias generaciones de egresados - la primera egresó en 2009- se han convertido en actores regionales y comunitarios de gran relevancia, poniendo de manifiesto su versatilidad y su capacidad de incursionar en múltiples ámbitos, locales y extra-regionales, profesionales y académicos.

Una ruta elegida por egresados de la uvi es ingresar a posgrados en México y otros países, tanto de investigación (por ejemplo, la Maestría en Investigación Educativa de la Universidad Veracruzana), como profesionalizantes (por ejemplo, la Maestría Internacional en Práctica del Desarrollo y la Conservación del Centro Agronómico Tropical de Investigación y Enseñanza en Costa Rica). En la mayoría de los casos de egresados cursando posgrados, se observa que eligen programas con afinidad o cercanía con el trabajo de campo en el medio rural e indígena, con enfoques participativos y/o con la interculturalidad como objeto de estudio. En el acompañamiento etnográfico a egresados de la UVI que se ha realizado a partir del proyecto InterSaberes, se ha documentado que: “La Uvi parece lograr 'cualificar' sus procesos migratorios posteriores: los egresados ya no emigran a la ciudad como jornaleros o peones, sino como becarios y futuros docentes o investigadores" (Dietz, Mateos y Mendoza, 2016: 821). A partir de su formación con enfoque intercultural, “[...] las y los egresados de la UVI interrelacionan los conocimientos académicos y/o 'científicos', recibidos durante su paso por la institución, con aquellos adquiridos en la cotidianidad, conocimientos prácticos y/o locales tales como la experiencia dentro de la vida familiar o comunitaria" (Dietz, Mateos y Mendoza, 2016: 822). Así, los egresados son portadores de saberes-haceres, que son "[...] saberes prácticos, aplicados, que se han obtenido en el contexto educativo. Resultan de la aplicación de lo aprendido en el aula, de la combinación de lo teórico y práctico" (Dietz, Mateos y Mendoza, 2016: 822).

La continuación de su formación académica conlleva complementar sus saberes-haceres, y como ellos mismos dicen, una ampliación de sus horizontes en términos de saberes académicos que les resultan novedosos. Sin embargo, esto ocurre en el marco de tensiones con saberes que prevalecen en el 
curriculum de los programas que cursan y en las prácticas de investigación convencionales - no interculturales - que ahí prevalecen.

Para explorar lo anterior, este artículo está organizado de la siguiente manera. Después de presentar la aproximación teórica a los saberes-haceres y saberes académicos, la metodología seguida para la construcción y el análisis de datos y el contexto de la formación universitaria con enfoque intercultural, los resultados se exponen en dos partes: 1) un balance retrospectivo de los egresados sobre sus saberes-haceres y 2) las tensiones que se producen en sus estudios de posgrado cuando sus saberes-haceres se ponen en contacto con saberes académicos, pero también los aprendizajes que complementan sus saberes previos al introducirse en sus nuevas comunidades académicas.

\section{Saberes-haceres y saberes académicos}

La formación de profesionales en la UVI — en el marco de la Licenciatura en Gestión Intercultural para el Desarrollo - se orienta a la mediación que se realiza entre comunidades y regiones rurales e indígenas con instituciones, organizaciones, entidades y actores externos de diferentes ámbitos. La investigación vinculada es la forma más común en la UVI para nombrar los procesos de gestión en los que la generación de conocimiento sobre y para los procesos comunitarios y locales es considerada importante. Las competencias que desarrollan los estudiantes de la uvi han sido objeto de estudio en el proyecto que enmarca este artículo (Dietz y Mateos, 2015; Dietz y Mateos, 2016; Mateos, 2015), y para ello se ha recurrido a la noción de savoir-faire, de "saber-hacer", desarrollada por Chamoux (1987) para referirse a los procesos profesionales de artesanos nahuas. Partimos de reconocer que saber algo significa realizarlo (Bermúdez Guerrero, 1995). En este sentido, los sabereshaceres no separan tajantemente teoría y práctica, sino que la articulan, y se orientan tanto a la acción como a la generación de conocimiento partiendo de "otras" epistemologías (Olivé, 2009). Esto es escasamente reconocido en el ámbito académico de los posgrados que cursan los egresados y en otros, por ejemplo, en el diseño y la implementación de programas sociales que, "desde arriba", desdeñan los saberes de "la gente" por su carácter "empírico"; como lo propone De Zutter (1997) a partir de la capitalización de experiencia como estrategia del posicionamiento de los saberes locales, “[...] no se trata de buscar la destrucción del sistema de saber dominante sino de bajarlo de su pedestal y traerlo a la mesa de negociación y concertación para emprender la recomposición del saber que el mundo actual tanto necesita" (p. 35). Se trata también de derribar la idea de que los saberes atemporales, aespaciales y 
abstractos que han sido posicionados por la ciencia, la academia y las grandes agencias internacionales de desarrollo (Cox, 1996) son superiores a los saberes locales concretos que forman parte fundamental de los saberes-haceres de los cuales nos ocupamos en este artículo. En el mismo sentido, Mato (2008) propone una colaboración intercultural en la producción de conocimientos - y de saberes-haceres, agregaríamos - que supone el reconocimiento de múltiples formas de producción, de acción, dependiendo de los diversos contextos.

En esta línea, se analizan saberes-haceres que se desarrollan en la UVI y las formas de producirlos, atendiendo a contextos, temporalidades, espacios e intencionalidades políticas diferenciadas. Al egresar, los gestores interculturales para el desarrollo lidian con tensiones cuando sus saberes-haceres intentan entrar en diálogo con otros tipos de saberes, que pueden ser caracterizados como académicos. El diálogo de saberes que entraña una ecología de saberes (Santos, 2006) enriquecida por la convivencia de prácticas heterogéneas de actores, que no son simples portadores de saberes estáticos sino productores de nuevas formas de comprensión (Leff, 2004), constituye una aspiración y un proceso; los intentos por concretarlo ocurren en medio de tensiones y estrategias para dilucidar su complementariedad y sus sinergias.

Los egresados que optan por estudiar posgrados se enfrentan a procesos de intermediación sui generis: entre sus propios saberes-haceres y esos saberes académicos que el curriculum de posgrados y las prácticas de investigación no interculturales enarbolan. Este ámbito de intermediación ha sido poco explorado y es precisamente del cual nos ocupamos en este artículo.

Los saberes-haceres de los egresados se construyen y fortalecen durante su estancia en la UVI, a partir del reconocimiento del pluralismo epistemológico (Olivé, 2009), poniendo en relación los conocimientos académicos que adquieren en su licenciatura y aquellos desarrollados en su vida cotidiana, en la familia y la comunidad, y que están conectados a sus prácticas cotidianas (Urrieta, 2013). Si bien estos últimos son visiblemente prácticos, la distinción teoría-práctica debe problematizarse y reconocerse que ha contribuido a jerarquizar tipos de saberes confiriendo superioridad al conocimiento que es producto de la aplicación del método científico en el marco de instituciones eurocentristas, etnocentristas y racistas que tienen su monopolio como las universidades no interculturales. Es relevante mencionar que los mismos egresados reproducen esta dicotomía "teoría-práctica" y se refieren a sus saberes comunitarios y locales como "prácticos" e incluso a muchos de los conocimientos adquiridos en la UVI, y, por otro lado, se refieren a la "teoría" como aquel cúmulo de conocimientos que emanan de procesos de investigación científica y de las disciplinas académicas con las cuales entran en contacto en sus posgrados. 
Sin embargo, nos interesa enfatizar que la producción de teoría ocurre dentro y fuera de la escuela y de la academia; ocurre al nombrar, al clasificar, al relacionar, es decir, está imbricada en el uso mismo del lenguaje. Además, es preciso decir que la distinción entre saber y el conocimiento está también relacionada con procesos de jerarquización epistemológica que no tenemos la intención de reproducir; por ello, al referirnos a saberes y a conocimientos, lo hacemos sin suponer una jerarquía, sino más bien a un uso generalizado para aludir a lo académico como conocimiento, y a lo local y cotidiano como saber. Quintriqueo y Torres (2012) se refieren a la distinción entre saber propio y conocimiento occidental disciplinar en las escuelas mapuches - aplicable a otros contextos indígenas como el abordado en este artículocoexistiendo y poniéndose en juego en la formación escolar. De este modo consideramos que si bien los saberes-haceres tienden a estar conectados con su aplicación, con las prácticas cotidianas de sus portadores, éstos tienen un componente teórico que bien puede emanar de la academia y la escuela, pero también de la sabiduría local.

Partiendo de lo anterior, nos interesa profundizar en aquellos saberes-haceres que resultan del contacto y el intercambio entre saberes comunitarios no escolares y saberes académicos aprendidos en la UVI, y que constituyen "otro" tipo de saberes cuando se llevan a contextos académicos no interculturales, como los programas de posgrado en los que se están formando los egresados entrevistados y que enfatizan el saber asociado a disciplinas científicas occidentales (Pérez y Argueta, 2011), como por ejemplo, la Maestría en Lingüística Indoamericana del Centro de Investigaciones y Estudios Superiores en Antropología Social en México y la Maestría en Comunicación Social de la Pontificia Universidad Católica de Chile.

Estos egresados de la uvi conocen formas "otras", novedosas - generalmente no valoradas - de hacer investigación en programas eminentemente académicos. Esto produce tensiones en el intento de los egresados de articular sus saberes-haceres a saberes académicos. Pero a su vez esos saberes académicos con los que entran en contacto complementan su formación. En el proceso formativo se desarrollan tensiones y aprendizajes que complementan saberes previos que contribuyen en la formación de los egresados cuando intentan poner a dialogar sus saberes-haceres fuera de sus contextos comunitarios y de programas académicos con enfoque intercultural. En este sentido, las tensiones y los aprendizajes que complementan saberes previos también constituyen dimensiones de un proceso que se visibiliza en universidades y programas académicos donde estudiantes de universidades interculturales desarrollan saberes-haceres en sus procesos de investigación. Dichos saberes-haceres ya no se consideran objetos de investigación sino herramientas 
epistemológicas, teóricas y metodológicas para generar conocimiento, y también cuestionar y transformar el status quo.

\section{Metodología}

Si bien el acompañamiento etnográfico doblemente reflexivo (Dietz, 2011) a los egresados se ha realizado en el marco del proyecto InterSaberes, los datos que se han obtenido para el análisis de las tensiones y oportunidades de egresados de la uvi en sus e-studios de posgrado derivan de entrevistas semi estructuradas y en profundidad que se realizaron a once egresados - seis hombres y cinco mujeres - de las cuatro sedes de la uvi que han continuado estudios de posgrado en programas nacionales y en el extranjero. Dichos egresados fueron identificados a partir del contacto establecido a través del proyecto InterSaberes y de información proporcionada por la UVI.

Todos son egresados de la LGID de las cuatro sedes regionales (tres de Grandes Montañas, cuatro de Las Selvas, dos de la Huasteca y dos del Totonacapan) y la mayoría ha continuado sus estudios en el ámbito de las Humanidades y en disciplinas relacionadas con su orientación dentro de la LGID (como la lingüística, la sustentabilidad, la comunicación, etc.), pero también en el ámbito educativo (tanto en posgrados de investigación y profesionalizantes donde lo intercultural es tematizado, y no constituye un enfoque a partir del cual se impartan dichos programas). Ocho egresados entrevistados estudiaron en México, y tres lo hicieron en el extranjero (Chile, Costa Rica y Ecuador).

En las entrevistas el propósito fue indagar sobre su experiencia como estudiantes de posgrado considerando que en su formación en una licenciatura con enfoque intercultural se hacía énfasis en la investigación vinculada a la transformación y la incidencia en la solución de las problemáticas de sus comunidades. Las preguntas apuntaban a la valoración de ambas experiencias de formación - licenciatura y posgrado - y a la reflexión sobre las tensiones que se les presentaron en sus procesos de investigación en el posgrado, así como la oportunidad de complementar sus saberes-haceres profesionales.

Los ítems más importantes se refirieron a: los aspectos más significativos de los dos procesos formativos; el contraste y la complementariedad entre la formación con enfoque intercultural en la UVI y en el posgrado; el abordaje teórico y metodológico de sus procesos de investigación en la licenciatura y el posgrado; la determinación de relevancia y la utilidad de los procesos de investigación; las tensiones en el tránsito de un modelo intercultural en licenciatura a uno convencional - es decir, no intercultural - en el posgrado, así 
como las estrategias para atenderlas; las fortalezas y las debilidades de cada uno de los procesos formativos.

Los elementos iniciales para el análisis de datos derivan directamente de las preguntas incluidas en las guías de entrevista. Un segundo momento se analizaron dos categorías fundamentales, de las cuales derivan sub-categorías identificadas inductivamente en el discurso de los entrevistados. La primera categoría se refiere a saberes-haceres construidos en la UVI, que se desglosa en varias sub-categorías: 1) sensibilidad para responder a necesidades locales y comunitarias a través de proyectos participativos e inspirados en prácticas locales, 2) trabajo de campo con métodos etnográficos, 3) diseño de protocolos metodológicos respetuosos y horizontales, 4) reconocimiento/valoración/descripción de saberes locales y 5) diseño de proyectos con objetivos de transformación social. La segunda categoría se refiere a saberes aprendidos tanto en la UVI como en los posgrados, que se complementan y también entran en tensión; ésta se divide en las siguientes sub-categorías: 1) encuentro con "lo teórico" o saberes-saberes, 2) crítica y reflexión sobre los procesos de investigación/intervención, 3) formas de incidir más allá del ámbito comunitario, 4) diseño y ejecución de proyectos de investigación a corto plazo/transformadores a largo plazo, 5) encuentro con disciplinas académicas, 6) aprendizaje de saberes del investigador y 7) desconocimiento de saberes no académicos y de sus portadores. A partir de estas dos categorías y sus correspondientes sub-categorías se estructuró este artículo.

\section{Contexto de la investigación}

Nos parece importante presentar el contexto de la investigación desde la voz de los egresados de la UVI, haciendo visibles las condiciones en las que han transitado por la escuela. Los estudiantes de la UVI han recorrido, en su mayoría, una trayectoria escolar precaria por las condiciones de la educación básica y media superior en los contextos rurales donde viven. Muchos de ellos estudiaron en telesecundarias y telebachilleratos, que son las modalidades más marginales de dichos niveles educativos. La precariedad de sus trayectorias es un aspecto que la mayoría de los entrevistados resalta como una característica de su formación académica y se refieren a ella cuando hablan de sí mismos como sujetos escolarizados en el medio rural. Esto se complejiza cuando consideramos que algunos de ellos son bilingües en alguna lengua indígena y en español (teniendo como lengua materna cualquiera de las dos) y que su lengua indígena es generalmente desplazada en los procesos 
educativos. A eso se añade una característica más a su experiencia escolar: el desdén por sus saberes comunitarios.

Considerando que las anteriores constituyen experiencias trascendentales para los jóvenes que ingresan a la UvI, su acceso a la licenciatura constituye un parteaguas a niveles individual, familiar y comunitario, detonante de múltiples procesos personales y colectivos.

La UVI se orienta a formar nuevos perfiles requeridos en las zonas indígenas para contribuir a su transformación, partiendo de las propuestas e iniciativas de los actores regionales y haciendo énfasis en construir ambientes interculturales donde las lenguas indígenas se fortalezcan y sus prácticas culturales sean valoradas, esto a partir de la intención de forjar una nueva relación con saberes no académicos e inspirada en un pluralismo epistemológico. Algunos egresados mencionan que si comparan la licenciatura con los programas de posgrado en los que estudiaron, destaca su flexibilidad para realizar proyectos de investigación con un amplio abanico de temáticas y métodos ante realidades diversas y cambiantes, e incluso respondiendo a peticiones concretas por parte de actores regionales. Sus egresados se forman en el hacer de procesos de investigación vinculada a la gestión intercultural, entendida como una práctica de mediación que posibilita iniciar y/o fortalecer procesos con la participación de los actores involucrados. Así:

[...] en tanto liga la producción de conocimiento a los procesos regionales y se articula en torno a la necesidad de mejorar las condiciones de vida de los habitantes, tiene como eje de su praxis la gestión intercultural, como motor de transformación, vinculación y mediación entre actores, saberes y culturas. La investigación con estas características aspira a fortalecer los procesos de gestión, al tiempo que éstos se retroalimentan del conocimiento generado en la investigación (UVI, s/f: 1-2).

Este tipo de investigación sui generis privilegia el desarrollo de saberes-haceres que tienen que ver con gestión, colaboración, organización, animación, derivando en un trabajo de campo que combina lo participativo y lo etnográfico, etc., y en menor medida con procesos de investigación documental, abstracción y teorización de procesos que se observan o estudian, que podrían considerarse "saberes-saberes". Esta aproximación a las comunidades busca distanciarse de prácticas extractivistas que sólo persiguen generar conocimiento, sin una articulación concreta a problemáticas sentidas por los actores comunitarios. Así, la investigación vinculada que propone la UVI marca distancia de las prácticas de investigadores que históricamente han visitado las comunidades y de las cuales se tiene memoria y se reflexiona en el ámbito comunitario. Un egresado apunta a la necesidad de una educación y una 
investigación vinculadas a la reflexión y la transformación de la realidad que los pueblos indígenas viven:

[...] ¿en qué medida todo esto que he aprendido hasta el momento en verdad me es útil para transformar la realidad y no sólo para academizar las igualdades y documentar todas las crisis actuales que se viven en este país? Y esto último justamente representa mi principal crítica al papel de la educación frente a las desigualdades y las injusticias, porque si la educación no sirve para mejorar las condiciones de vida de la población, sobre todo de los más desfavorecidos, entonces algo no está bien y debe ser replanteado (E2).

Sin embargo esto constituye una forma muy específica de investigar que no se ha trasladado a licenciaturas no interculturales. La distancia en términos epistemológicos, metodológicos y políticos con procesos de investigación en posgrados puede ser abismal. A eso se enfrentan los egresados de la Uvi cuando ingresan a estudios de posgrado "no interculturales". Los entrevistados han optado por una diversidad de programas. La mayoría elige programas nacionales y pocos cursar otros en el extranjero; tres estudiaron su posgrado en Latinoamérica, concretamente en Chile, Ecuador y Costa Rica. Un aspecto relevante de las características de los posgrados elegidos es que algunos han sido con enfoque en investigación y otros con carácter profesionalizante. Han cursado posgrados en disciplinas cercanas a alguna de las orientaciones por las que optaron en la LGID - como lingüística, antropología visual, comunicación, agroecología - ; en programas en los que lo intercultural constituye un objeto de estudio o que son afines a su formación con enfoque intercultural - sobre educación intercultural, estudios interculturales y en educación para la interculturalidad y la sustentabilidad - . Sin embargo, ninguno de los posgrados tiene propiamente un enfoque intercultural.

Los egresados contrastan la valoración y la utilidad de los saberes-haceres que portan, tanto en la Uvi como en sus estudios de posgrado; y por otro lado contrastan el vínculo que se establece entre la educación y la investigación que realizan y las realidades que se estudian. En este contraste, se recurre a dicotomías tales como "saberes tradicionales/saberes científicos", "práctica/teoría" e "investigación vinculada/investigación científica", por mencionar algunas. Asimismo, surgen preocupaciones sobre la dimensión política y la relevancia social de la investigación y los procesos de escolarización. En relación a lo anterior, a continuación exponemos un análisis en dos partes: 1) un balance retrospectivo de los egresados sobre sus saberes-haceres construidos en la universidad y 2) las tensiones que se producen en sus estudios de posgrado "no interculturales" cuando sus saberes-haceres se ponen en 
contacto con saberes académicos, pero también las sinergias que se produce cuando sus saberes-haceres previos se integran en sus nuevas comunidades académicas.

\section{Balance retrospectivo de los egresados sobre sus saberes-haceres construidos en la UVI}

Los egresados realizan una valoración de los saberes locales y no escolares que portan como miembros de sus comunidades y que cobraron un sentido distinto a partir de su posicionamiento en los procesos educativos de la UVI para interrelacionarse con los saberes académicos. En ese sentido, valoran los conocimientos que circulan entre todos los actores comunitarios y que son construidos a partir de procedimientos y metodologías distintos a las científicos. La colectividad es portadora de saberes, como lo menciona un egresado:

[...] el conocimiento de la comunidad no sólo lo tienen los sabios, lo tiene toda la comunidad. Es un conocimiento que se comparte, que se transmite de generación en generación y se expande a todas las familias. Entonces cada uno tiene una especialidad, pero casi todos son transdisciplinares (E1).

En este mismo sentido, la investigación de y sobre lo propio conduce a un aprendizaje, y más aún, a la posibilidad de avanzar a la decolonialidad del propio ser (Maldonado-Torres, 2009).

[...] cuando estuve en la comunidad nunca dejé de aprender, cada encuentro, cada espacio de diálogo me sirvieron para descolonizarme a mí, en mi propia historia, no sé en qué medida, pero estoy seguro de que mi paso por la comunidad me volvió más humano, más sensible y yo también estoy absolutamente convencido de que fui a la comunidad a aprender y no a enseñarles nada (E2).

Resalta la importancia que los egresados se atribuyen a ellos mismos como portadores de saberes comunitarios, los cuales son considerados como "objeto de estudio" por investigadores de disciplinas con los que entran en contacto, como saberes susceptibles de ser analizados desde una perspectiva científica, generalmente desde la antropología o las llamadas etnociencias (Pérez y Argueta, 2011). Esos saberes comunitarios desdeñados durante casi toda su trayectoria escolar se reposicionan, como lo menciona un egresado: 
Mi fortaleza es que soy de una comunidad. A veces sentía que los investigadores discuten sobre lo que yo soy, hasta en la misma maestría o en la misma UVI dicen: "las comunidades son tal, las comunidades no saben". Entonces como yo soy la comunidad, conozco la comunidad, me formé entre la comunidad, eso es lo que me da confianza y fortaleza (E1).

La lengua indígena, como saber propio, se reposiciona en los procesos de investigación vinculada, como lo afirma un egresado bilingüe de español y náhuatl:

Definitivamente hablar, escribir y conocer bien mi lengua materna ha sido una ventaja en mi proceso formativo. Y no porque haya sido vehículo en el proceso de enseñanza aprendizaje, sino porque me sirvió en mi proceso de intervención, a mi trabajo con las personas con quienes colaboré, y lo que también me sirvió fue conocer bien mi comunidad, sus dinámicas y la región (E2).

Estos saberes propios, locales, comunitarios, extra-escolares se convierten en capital en los procesos de investigación vinculada, forjándose así saberes-haceres que distinguen a los egresados del programa con enfoque intercultural de la uvi. La identificación de sus propios saberes-haceres, durante o después de haber estudiado un posgrado, los lleva a realizar un balance en el que sobresalen cinco saberes-haceres que consideran relevantes en su nueva etapa formativa en posgrados no interculturales y que se analizan a continuación.

El primer saber-hacer que los egresados consideran relevante es la sensibilidad para responder a necesidades locales y comunitarias a través de proyectos participativos e inspirados en prácticas locales. La participación, la relevancia y la pertinencia son tres elementos que en la UVI se presentan como constituyentes de la investigación vinculada. Se definen en los siguientes términos:

Participación: La investigación promueve la participación para el mejoramiento de las condiciones de vida de los habitantes de las regiones de impacto, por lo cual debe buscar en todo momento la colaboración de actores de los pueblos originarios directamente relacionados con el/ los problemas de investigación, en su delimitación, su desarrollo y su evaluación [...]

Relevancia y pertinencia: el proyecto es relevante y pertinente en regiones donde tiene presencia la UVI, entendiendo la relevancia en su potencial transformador para la atención de los problemas que aquejan las regiones y las iniciativas que emerjan de ellas. Los proyectos deben buscar en todo momento la generación de conocimiento pertinente a la vida so- 
cial de los pueblos originarios. Las comunidades y los individuos directamente involucrados en el proyecto deben tener voz y participación en la formulación del mismo, de manera que se asegure, en todo momento, que los proyectos de investigación sean insumos para el mejoramiento de la calidad de vida y del buen vivir de los pueblos originarios, en esta medida serán pertinentes y contribuirán a la construcción de una vida digna [...] (UVI, s/f: 3-4).

Lo anterior busca el desarrollo de un saber-hacer para detectar necesidades y desarrollar proyectos que se orienten a su solución de forma participativa. Desde una lógica local y/o comunitaria, dicho saber-hacer es reconocido por los egresados como un valor, no sólo metodológico sino actitudinal.

El programa busca desarrollar destrezas y actitudes que me permitieron ser sensible a las necesidades a nivel local y proponer de manera conjunta con los actores sociales. La educación intercultural en la UVI me cambió, sembró actitudes, por ejemplo, la sensibilidad, la visión en busca del progreso a nivel comunitario y lograr incidir a nivel comunitario, buscar un cambio social en mi propia comunidad (E4).

Este saber-hacer implica también definir proyectos desde otra lógica, que no es la académica ni objetiva, y que involucra a las comunidades. Es relevante cuando un egresado habla de necesidades y problemáticas "sentidas" por las comunidades y del "acompañamiento" del gestor. Aquí resalta la dimensión de lo sentido y no definido objetivamente y "desde afuera", apuntando a nuevas formas de comprensión en el sentido que Leff (2004) propone al hablar de diálogo de saberes. Así lo menciona un egresado:

En la uvi se enseña a los estudiantes a trabajar sobre necesidades y problemáticas concretas que son sentidas por las comunidades y que éstas no tienen que ser delimitadas objetivamente, ni a través de la formulación y la comprobación de hipótesis, sino más bien atendidas a través de proyectos de intervención-acompañamiento como yo los llamo, que involucran la participación activa de las comunidades (E2).

Para profundizar en ello, la comunicación en lengua indígena como una práctica cotidiana, en espacios participativos, que no se conciben como espacios de "recolección de datos" al estilo extractivista, se considera crucial. Una egresada bilingüe en español y náhuatl revalora su lengua materna diciendo:

Aprendí a observar paulatinamente a las personas e identificar las necesidades que tienen en la comunidad, comunicándonos desde la lengua para generar confianza, apoyando en las necesidades que tienen en 
cuanto a la salud $[. .$.$] a través de talleres donde ellas pudieran opinar,$ participar y conocer las necesidades para poder intervenir en la problemática (E5).

Este saber-hacer permite que la investigación vinculada sea pertinente para la toma de decisiones en las comunidades y/o sus grupos, porque considera solicitudes expresas, y en este sentido, se torna útil. La pertinencia de los proyectos es el motor para la colaboración de los actores sociales a los que interpelan. Así lo afirman dos egresados:

Que la investigación o proyecto responda a las necesidades del grupo con el que vinculamos, puede ser en nuestra comunidad, en una comunidad distinta, con productores, con padres de familia o diferentes grupos. Yo creo que todo trabajo es relevante, si no fuera relevante, no haría que los mismos actores de la comunidad encaminen el proyecto (E1).

Una investigación es exitosa, creo yo, sólo si sirve y responde a las necesidades de las personas con quiénes colaboras, si no hay que replantearlo (E2).

Un segundo saber-hacer que complementa la perspectiva de investigación vinculada de corte participativo desde el punto de vista de los egresados ha sido la conducción de trabajo de campo a través de métodos etnográficos. Su preeminencia tiene que ver con la formación de sus docentes y de quienes diseñaron el programa de licenciatura (varios de formación antropológica). En este sentido, son los egresados que han incursionado en maestrías en investigación quienes enfatizan este saber-hacer, que no es precisamente el de un antropólogo, sino el de un actor comunitario que trabaja con colaboradores para el desarrollo de un proyecto que puede tener un componente investigativo y de diagnóstico que fundamente la intervención, para el que estos métodos son utilizados con matices específicos. Una egresada que en su maestría realiza trabajo etnográfico menciona:

[...] la etnografía yo la estuve trabajando desde la UVI, cómo acercarte con las personas que vas a trabajar; yo creo que esa parte de la Uvi a mí me ha ayudado mucho [...] la experiencia previa que yo traigo de la UVI de trabajar con la gente, aprender hacer una entrevista semiestructurada, el aprender a relacionarme (E6).

La incorporación del uso de métodos etnográficos detona un saber-hacer que pone énfasis en la construcción de datos empíricos de corte cualitativo desde una visión cercana y directa de problemáticas en las que se interviene. 
Un tercer saber-hacer fundamental en contextos indígenas a nivel mundial en el que la investigación ha sido históricamente una experiencia violenta y extractivista propia del colonialismo (Smith, 2002), fue el de diseñar protocolos metodológicos respetuosos y horizontales para el acceso a sujetos y saberes, así como al uso y la publicación de los datos empíricos. En el caso de los estudiantes de la UVI, fue necesario desarrollar un saber-hacer que considerara de forma central la anuencia negociada - partiendo de parámetros propios - con las comunidades y los actores. Un egresado menciona al respecto:

Si nosotros estamos trabajando en contracorriente de una investigación convencional, del extractivismo, nosotros tenemos que preguntar a la comunidad, a los actores que sí se puede escribir sobre lo que estamos viendo, que si nos dan el derecho de poder compartir con los demás sus saberes [...] en las entrevistas que vamos haciendo no es para recolectar, sino que es para generar un campo de diálogo, de reflexión entre entrevistado y entrevistador y en el que en conjunto salen nuevas ideas, nuevos conocimientos, nuevas formas de pensar. Entonces ellos son con los que tenemos que hacer un diálogo, tenemos que hablar, hacer acuerdos para poder para dar a conocer los conocimientos que se generan en el grupo, o que una comunidad desarrolla con sus integrantes (E1).

Un cuarto saber-hacer fundamental para los entrevistados es la identificación de saberes locales para su descripción, su reconocimiento y su valoración; lo hacen reconociendo que los saberes locales se transmiten entre generaciones y pueden ser posicionados en el curriculum de todos los niveles educativos, como el universitario, así como en los procesos que ahí se desarrollan. Todos enfatizan su capacidad de reconocer y valorar saberes comunitarios que fueron desdeñados e invisibilizados en las escuelas y otras instituciones. Ese desdén aisló dichos saberes, pero su formación intercultural contribuyó a una reconexión con ellos a partir de su identificación y su puesta en práctica. Una egresada menciona al respecto:

Los saberes son un conocimiento que se genera en la comunidad que vive a diario con estas cosmovisiones. Y para las personas es válido este conocimiento, porque entienden a profundidad los saberes que se viven en un pueblo; y no podemos decir que conocemos los saberes que hay en nuestra comunidad cuando no los hemos puesto en práctica; sólo nuestros padres, tatarabuelos, conocen estos saberes (E5).

Este testimonio apunta a la comprensión de dichos saberes locales a partir de su práctica y de su validez a partir de un conocimiento profundo. El reconocimiento de dichos saberes ignorados por nuevas generaciones, su valora- 
ción a partir del encuentro con un enfoque intercultural que los reposiciona y su sistematización permiten que sean visibilizados por los mismos actores comunitarios y otros externos.

Un quinto saber-hacer considerado clave por los egresados de la UVI es el diseño de proyectos con objetivos de transformación social. La intención de transformar el status quo de las comunidades, partiendo de diagnósticos como parte de su formación profesional, pero también de la propia vivencia de la marginalidad de las zonas indígenas y rurales donde habitan, es común a los entrevistados. En este sentido una egresada dice: “[...] en la uvi a veces pensamos mucho en la transformación, en que nuestros propios proyectos puedan llegar a fortalecer ciertos procesos" (E6).

Ese énfasis revela que los alcances de la transformación son valorados por los egresados en retrospectiva; en ocasiones son matizados y moderados por una reflexión que es mediada por nuevos referentes y criterios considerados en sus posgrados para el desarrollo de proyectos en los cuales la transformación puede no ser central.

En el balance de los egresados, los cinco saberes-haceres arriba mencionados son referidos como los más relevantes de su etapa formativa en la Uvi; sostienen que lo siguen siendo en sus estudios de posgrado. Sin embargo, la relevancia de dichos saberes-haceres no está exenta de tensiones, aunque, por otra parte, se detona un proceso de diálogo de saberes incipiente, también fructífero, en el contacto con saberes relacionados con una variedad de disciplinas, epistemologías y metodologías "convencionales", es decir, no interculturales.

\section{Egresados de la UVI en posgrados no interculturales: ampliación de horizontes y tensiones}

¿Qué sucede con egresados portadores de saberes-haceres no académicos cuando ingresan a posgrados no interculturales? En todos los casos, emerge la idea de la complementariedad de sus saberes-haceres previos, de sinergias, de una ampliación de sus horizontes, sobre todo de carácter teórico, pero también de haber experimentado tensiones importantes al desarrollar sus proyectos a partir de sus saberes-haceres previos o al intentar posicionarlos.

Es importante señalar que los posgrados elegidos por los egresados tienen en su mayoría una orientación disciplinar: antropología, comunicación, lingüística, agroecología, etc., con tradiciones epistemológicas, teóricas y metodológicas a las que han requerido adentrarse. En ese encuentro surgieron tensiones importantes, pero que finalmente se convirtieron en aprendizajes 
que complementaron sus saberes-haceres. A continuación se presentan tensiones y aprendizajes complementarios relevantes para los egresados de la UVI en posgrados "no interculturales".

El encuentro con lo teórico o saberes-saberes constituye para todos los entrevistados una fuente tanto de tensión como de sinergia. Esto lo vinculan a un déficit en el abordaje de conceptos y teorías en su licenciatura. Lo relacionan con los "saberes-saberes" que son conocimientos de carácter académico, asociados a lo pedagógico-áulico, abstractos y descontextualizados (Dietz, Mateos y Mendoza, 2016: 813). En ese sentido, argumentan que con el posgrado logran complementar sus fortalezas en la construcción de datos y la intervención con grupos, al observar procesos más orientados a lo teórico. Así lo narra una egresada que cursa un posgrado con orientación a la investigación:

[...] en el posgrado la parte teórica queda más cargada. En la uvi más la parte empírica queda muy fortalecida porque nos llevamos mucho tiempo en los propios procesos de intervención y luego a la parte teórica falta como dedicarle un poco más de tiempo. Esa es una gran diferencia entre un proceso convencional y uno intercultural; vas aprendiendo más con lo que hacías en campo, por la parte empírica, y la maestría es como un poco de los dos, pero rebasa la parte descriptiva, sino que incluye interpretación, reflexión, traducción (E6).

El encuentro con lo teórico en los posgrados no interculturales constituye una ampliación de horizontes, pero también una tensión importante al asumirse como debilidad de la LGID. En este sentido, un egresado menciona al respecto:

En la maestría me di cuenta de la importancia que tiene la teoría, y de hecho los posgrados están más enfocados justamente en esta parte, en la teorización, en lo abstracto, pero sobre todo en la producción científica. Yo admito que cuando llegué a la maestría no tenía tantas herramientas teóricas como las que tengo ahora, porque había trabajado mucho de cerca con las comunidades, pero no manejaba tantos conceptos, y bueno, me costó un poco teorizar, pues se trata de un ejercicio complejo y esquizofrénico, diría yo. Una necesidad es estar leyendo y teorizando constantemente, y bueno, en la UVI no había ejercitado tanto estos procesos, y ésas considero fueron mis mayores debilidades, temores en ambos procesos (E2).

Una segunda fuente de tensiones se origina en el énfasis en lo empírico y el trabajo de campo que los egresados tuvieron durante la formación en la UVI y que se tradujo en poco énfasis en el abordaje de teorías a partir de las cua- 
les reflexionar y analizar sus diagnósticos e intervenciones comunitarios. A decir de los egresados, en el marco de la LGID, los procesos se sistematizaban de forma descriptiva, sin reflexión, crítica o análisis profundos. En cambio, en sus posgrados desarrollaron aprendizajes orientados a: “[...] analizar la cuestión teórica de manera más consciente, de manera más analítica, sin desapegarme de la cuestión comunitaria" (E7).

El posgrado se visualiza como el espacio formativo para adquirir nuevos saberes que permitan desarrollar un análisis crítico en los procesos de investigación, o generar sistematizaciones y capitalizaciones de la experiencia a partir de las intervenciones que vayan más allá de lo descriptivo (De Zutter, 1997). Así lo mencionan dos egresados:

En la licenciatura quizás se hacía investigación vinculada de una forma o tan profunda, y ya en la maestría es donde se pule y se empiezan a adquirir nuevas herramientas, a comprender, a entender mejor las herramientas o el tipo de metodologías que ocupamos, no para extraer información sino para istematizar experiencias que vamos generando con las comunidades (E1).

En este proceso de profesionalización uno va madurando y se va volviendo más crítico, y se va complejizando también nuestra visión de análisis de la realidad (E2).

Además de desarrollar un saber-hacer para un análisis crítico y reflexivo, consideran importante que vaya más allá del ámbito local y regional; en este sentido apuntan a un análisis que se ubique también en un plano nacional y mundial. Un egresado afirma:

Porque si a lo que se aspira es a continuar formándose, entonces esta parte es importante. No sólo analizar los fenómenos desde un contexto micro, más local como en el caso de la UVI, descuidando el análisis en contextos más globales. Los egresados de la uvi conocen muy bien las dinámicas, las problemáticas, las necesidades de sus pueblos, y tal vez eso mismo, desde una mirada más regional, pero no hay un proceso de reflexión, de teorización en contextos nacionales, y ya ni se diga mundiales (E2).

Una tercera fuente de tensiones y sinergias emerge cuando los egresados descubren otras formas de incidir más allá de lo comunitario. En los posgrados enfocados en investigación, los egresados se plantean proyectos que no incluyen la incidencia o la transformación en sus objetivos. Ese énfasis en la transformación social con y para los sujetos que consideran un plus, se desdibuja y pasa a ocupar un segundo plano u otras formas de incidir, como por 
ejemplo en el ámbito de las políticas públicas o en iniciativas desde el ámbito académico, por ejemplo, a partir del conocimiento indígena (OREALC/UNESCO, 2017). Un egresado que cursó un posgrado en investigación educativa menciona en este sentido:

Y entonces comprendí que una de las formas de incidir en la cuestión educativa y dentro del enfoque intercultural, si bien puede ser interviniendo en procesos comunitarios, en cuestiones organizacionales, con el programa de maestría podría incidir en proponer mejoras a determinados procesos académicos, generar política pública, iniciativas desde lo académico. Entonces yo encuentro que esas dos partes ayudaron a balancear mi parte personal comunitaria en la licenciatura, y en la maestría, la parte académica, investigativa, desde una mirada más holística y situada a los contextos donde suceden las problemáticas y necesidades de las comunidades (E7).

La naturaleza de los proyectos de los posgrados difiere de los que llevaron a cabo en la licenciatura en dos sentidos: 1) la incidencia para la transformación social no es un objetivo prioritario en el corto plazo ni como resultado directo de su desarrollo, y 2) la duración y la intensidad del trabajo de campo suele ser menor, ya que sus posgrados son escolarizados y ofrecidos por instituciones que no están en las regiones indígenas. Esto dificulta una estancia prolongada en campo y la creación y/o fortalecimiento de las relaciones con los actores, factores fundamentales para un proceso de investigación vinculada como el que desarrollaron en la UVI. La aspiración de los egresados es plantear transformaciones en sus comunidades a partir de procesos de investigación desde una lógica propia; en este sentido, Smith (2002) ha propuesto la creación de programas de investigación estratégicos para la justicia social y la reconstitución de las culturas indígenas. Un egresado que al cursar su posgrado desarrolló una tesis de corte etnográfico expone las tensiones entre proyectos eminentemente academicistas y otros que tienen su fundamento en las problemáticas de las comunidades:

[...] si los estudiantes tienen como informantes a actores con los que no se logra concretar ese trabajo a largo plazo, es decir, a darle seguimiento, porque los mismos tiempos administrativos y académicos del programa no te lo permiten, o sea, no hay como esa oportunidad de estar de manera continua con los actores que están compartiéndonos sus vivencias y sus saberes. Entonces ahí ese contraste, pues el programa intercultural tiende a ser un programa más cercano a las comunidades, mientras que la maestría tiende a ser un programa más academicista, riguroso en cuanto a las cuestiones conceptuales, las cuestiones metodológicas; y entonces profesionaliza al estudiante a tener conocimiento 
sólido en la investigación y entonces la cuestión comunitaria y actoral tiende a verse como los espacios donde se puede encontrar la información (E7).

Nos interesa resaltar el cambio en la concepción de los egresados sobre los actores y/o grupos que participan en proyectos de intervención como colaboradores a mediano y largo plazos, hacia la de sujetos que proveen información en proyectos de investigación de corto plazo. Por otro lado, se observa que el énfasis en la transformación no se mantiene dado el corto plazo de los proyectos de investigación de los posgrados no interculturales. Una egresada reflexiona lo siguiente al respecto: "Creo que en la uvi a veces somos muy ambiciosos, queremos hacer muchas cosas y nuestros objetivos son así, como que muy grandiosos, y no siempre se puede" (Е6).

Estos cambios son una cuarta fuente de tensiones y de sinergias. No siempre se capitalizan y se apropian como nuevas formas de proceder. En ocasiones, se confrontan y retan; y esto conlleva al cuestionamiento del posicionamiento de la investigación y la academia en el marco de la crisis social que vivimos. Un egresado narra lo siguiente a propósito de lo anterior:

Una vez uno de nuestros profesores en la maestría me dijo que eso era para lo que servían las tesis únicamente, para demostrar el dominio de determinadas variables teóricas en un proceso. Eso a mí me genera mucho ruido, me impactó mucho. Yo creo que no basta con analizar y documentar las múltiples problemáticas que afectan a los pueblos originarios, sino también trabajar para generar condiciones, aunque sea mínimas, que atiendan dichas problemáticas y que promuevan el desarrollo de las comunidades. Es importante que la academia asuma su papel de compromiso social con los sectores más desfavorecidos de este país, los indígenas, por ejemplo; que los investigadores se despojen de su investidura de supremacía y comiencen a establecer otro tipo de relaciones y de diálogos con las personas, con los individuos con quienes se colabora, pero unos más horizontales, más de iguales, más de compañeros (E2).

Una quinta fuente de tensiones y sinergias es el encuentro con las disciplinas académicas en los posgrados no interculturales. La UVI plantea una intención clara en trascender lo disciplinar - debido a que la gestión y el enfoque intercultural no se inscriben en una sola disciplina ni en la interdisciplinariedad - y orientarse a lo transdisciplinar:

La investigación se orienta por enfoques teóricos y conceptuales de tipo integral, transdisciplinar y complejo. Si bien se alimenta del conocimiento disciplinario, trasciende sus compartimentaciones teóricas (Alatorre, s/f), incorporando saberes no producidos desde las instituciones 
y tradiciones hegemónicas del conocimiento y generando propuestas epistémicas diversas que alienten la inclusión plural de actores, lenguas y conocimientos (UVI, s/f: 5).

Esto es valorado positivamente por los egresados, por la posibilidad de visualizar un panorama más amplio de lo investigado, como lo señala un egresado que cursó un posgrado en el extranjero:

Pues prácticamente yo iba con una mirada más intercultural, donde se valoran diferentes espacios, es más interdisciplinario; en la maestría me topé con compañeros que eran cineastas, antropólogos, que estudiaron comunicación, periodismo, artes, y cada quien como que iba con su propia mirada, desde el ámbito en que se desarrolló. Entonces me di cuenta que la UVI prácticamente te abre un panorama más amplio de cómo poder trabajar o tener una mirada más amplia y más holística desde el trabajo de campo (E9).

Sin embargo, también se genera una tensión cuando se insertan en programas disciplinarios y no con intenciones transdisciplinares asociadas al diálogo de saberes y a la transformación social (Pérez Luna et al, 2013). Así lo afirma un egresado:

En mi caso una necesidad que he detectado es mayor especialización en disciplinas duras como la antropología. Es una parte que no se atiende con relación a la formación en las disciplinas más duras y más convencionales. Pero si un estudiante quiere posteriormente ingresar a un posgrado en estas áreas definitivamente requerirá especializarse, profesionalizarse en estos ámbitos. Y eso es algo que me está ocurriendo a mí ahora en el empleo que tengo, y la manera de resolverlo es siendo autodidacta en todos esos temas (E2).

En este mismo sentido, los egresados asocian a los saberes propios de un investigador los siguientes: lectura, consultas bibliográficas, escritura académica, planteamiento de proyectos de investigación, entre otros. El énfasis de la UVI en una investigación de carácter vinculado que se centra en el trabajo con actores y/o grupos, y no en procesos de sistematización y análisis planteados por escrito, genera ciertos vacíos de los que los egresados se hacen conscientes cuando ingresan a los posgrados. Dos egresados reflexionan críticamente sobre este asunto apuntando a los saberes del investigador que requieren desarrollar:

No se inculca a un gestor intercultural a ser un buen investigador, o algo tan sencillo como leer; no se enseña a hacer consultas bibliográficas, no 
se termina de pulir esa destreza, y eso era lo que me hacía sentir débil, la falta de costumbre de leer, de escribir (Е4).

Lenguaje académico no se enseña. Recuerdo que desde el inicio a mí me costaba incluso elaborar un protocolo, porque yo lo estaba pensando más como en un proceso de intervención (E6).

Lo anterior tiene relación con dos aspectos: uno estructural, que se asocia al rezago educativo expresado por los mismos entrevistados y que no logra revertirse en la licenciatura; y otro relacionado con las características del documento recepcional - texto en el que los estudiantes de la uvi presentan el desarrollo del proyecto de intervención - entre las que destacan la sistematización de un proceso que no remite necesariamente a una reflexión teórica explícita, al planteamiento de un protocolo de investigación ni a la investigación bibliográfica intensiva.

Una última fuente de tensión proviene de la ausencia de reconocimiento de saberes que se han conceptualizado como no científicos y de sus portadores, por parte de académicos de posgrados no interculturales. Desde un pluralismo epistémico enarbolado por la Uvi, que resalta la importancia de "la construcción de conocimientos y saberes desde el reconocimiento, la convergencia y el intercambio entre miradas epistémicas diversas" (UVI, s/f: 5) y desde la transformación social, los egresados transitan a programas en los que la generación de conocimiento no se concibe en el marco que dicho pluralismo epistémico, y además, se encuentra distanciada de la incidencia para la solución de problemáticas concretas y de las preocupaciones de los actores sociales. Esta situación es referida por un egresado que cursó un posgrado con gran afinidad al enfoque intercultural:

No me ha tocado ver que un sabio local haya podido fungir como sinodal en un examen profesional, y eso sigue siendo este colonialismo interno a manera de crítica, entonces sobre el papel que debe jugar la academia en las investigaciones en relación a las problemáticas y múltiples crisis que aquejan a los pueblos originarios de este país en la actualidad, y que con el tiempo pues se agudizan cada vez más. Pienso que es muy importante cambiar el enfoque tradicional de investigar e intervenir en las comunidades, deben servir para algo más que demostrar el dominio de determinadas variables teóricas en un proceso (E2).

Los espacios académicos no interculturales no tienden al reconocimiento de saberes conceptualizados como no científicos ni de portadores de saberes locales que se construyen a través de otros procedimientos. Esto históricamente ha caracterizado dichos espacios por ser violentos y excluyentes de otros 
saberes y otras epistemologías (Smith, 2002). La vivencia de esta violencia epistémica (Spivak, 1988) es atestiguada por un egresado que desarrolló un proyecto en el que puso en el centro los saberes locales:

Yo reconocía que el aprendizaje más importante era el que había tenido con la comunidad, sus conocimientos; aprendí más de ellos que estando en la academia, pero también dije que eso no es posible en términos institucionales, que un sabio pueda fungir como director de una tesis, ni siquiera estar presente como sinodal. Y está claro, es otra forma de ejercer el colonialismo interno desde la academia, y marginar los conocimientos de los pueblos. Me ha tocado constatar que la academia y la investigación son los espacios sumamente excluyentes y violentos, más aún con las personas que provenimos de pueblos originarios (E2).

\section{Conclusiones}

El balance de los egresados resalta sus saberes-haceres construidos en la licenciatura entrando en tensión en su paso por programas de posgrado y, a su vez, su encuentro con otros saberes, sobre todo saberes-saberes, que complementan su formación previa en una universidad intercultural como gestores. Observamos que los estudios de posgrado constituyen una alternativa atractiva para egresados que buscan desarrollar saberes-haceres relacionados con la investigación - no precisamente vinculada - y/o especializarse en alguna disciplina afín a su orientación profesional (comunicación, derechos, lenguas, salud o sustentabilidad). Lo hacen intentando complementar sus saberes-haceres previos, pero también encontrando tensiones que los cuestionan.

La inmersión de los egresados de la UVI en una cultura académica no intercultural genera transformaciones en sus saberes-haceres en formas que no nos proponemos evaluar. A raíz de estos resultados, surgen nuevas interrogantes para futuras investigaciones en este novedoso campo. Nos preguntamos, en primer lugar, en qué medida la formación en posgrados no interculturales difumina/desplaza aquellos saberes-haceres de corte intercultural, colaborativo, horizontal y orientados a la transformación, para que prevalezcan otros de corte académico no intercultural que ignoran y/o cuestionan el pluralismo epistemológico, las metodologías colaborativas y objetivos orientados a la incidencia. Esto sin duda entraña retos para los programas de posgrado en términos de reconocer la riqueza que los estudiantes que provienen de licenciaturas con enfoque intercultural aportan al quehacer investigativo, y de no coartar diseños de investigación creativos en los sentidos antes descritos. 
En segundo lugar surgen preguntas sobre las formas en que los posgrados no interculturales y sus actores educativos (docentes, directores de tesis, estudiantes) capitalizan los saberes-haceres que portan los egresados de la UVI $-\mathrm{y}$ de otras universidades interculturales - en sus procesos educativos. Esos saberes-haceres portados por los egresados constituirían aportaciones de gran relevancia a los programas de posgrado no interculturales a los que ingresan. Si bien no visualizamos que transformen o cuestionen de forma profunda las bases epistemológicas y metodológicas a partir de las cuales funcionan dichos programas, su introducción podría generar preguntas y preocupaciones sobre el posicionamiento político de sus investigaciones, sobre el posicionamiento de saberes considerados "no científicos" en sus curricula e investigaciones, sobre el lugar que ocupan de los sujetos (e incluso en ocasiones "objetos") de investigación, entre otras.

\section{Referencias}

Bermúdez Guerrero, Olga María (2005). El diálogo de saberes y la educación ambiental. Bogotá: Universidad Nacional de Colombia.

Chamoux, Marie-Noëlle (1987). Trabajo, técnicas y aprendizaje en el México indígena. Ciudad de México: CIESAS.

Cox Aranibar, Ricardo (1996). El saber local, metodologías y técnicas participativas. La Paz: NOGUB-COSUdE y CAF.

Santos, Boaventura de Sousa (2006). Renovar la teoría crítica y reinventar la emancipación social. Buenos Aires: CLAcso.

De Zutter, Pierre (1997). Historias, saberes y gentes. De la experiencia al conocimiento. Lima: Escuela para el Desarrollo, Editorial Horizonte.

Dietz, Gunther (2011). Hacia una etnografía doblemente reflexiva: una propuesta desde la antropología de la interculturalidad. Revista de Antropología Iberoamericana, 6 (1), p. 4-26

Dietz, Gunther, Mateos Cortés, Laura Selene (2015). ¿Qué de intercultural tiene la" universidad intercultural"? Del debate político-pedagógico a un estudio de caso veracruzano. Relaciones. Estudios de historia y sociedad, 36(141), p. 13-45

Dietz, Gunther y Mateos Cortés, Laura Selene (2016). Universidades Interculturales en México. Balance crítico de la primera década. Revista Mexicana de Investigación Educativa, 21(70), p. 683-690

Dietz, Gunther, Mateos Cortés, Laura Selene, Mendoza Zuany Rosa Guadalupe (2016). ¿Saberes-haceres interculturales? Experiencias profesionales y comunitarias de egresados de la educación superior intercultural veracruzana. Revista Mexicana de Investigación Educativa, 21(70), p. 809-835

Leff, Enrique (2004). Racionalidad ambiental y diálogo de saberes, Polis [En línea], 7. 
Maldonado-Torres, Nelson (2009). Sobre la colonialidad del ser: contribuciones al desarrollo de un concepto. En Santiago Castro-Gómez y Ramón Grosfoguel (eds.), El giro decolonial. Reflexiones para una diversidad epistémica más allá del capitalismo global. Bogotá: Siglo del Hombre Editores, pp. 127-167.

Mateos Cortés, Laura Selene (2015). La formación de gestores interculturales: jóvenes profesionistas egresados de la Universidad Veracruzana Intercultural. Revista Interamericana de Educación de Adultos, 37(2), p. 65-81

Mato, Daniel (2008). No hay saber "universal", la colaboración intercultural es imprescindible. Alteridades, 18 (35), 101-116.

Olivé, León (2009). Por una auténtica interculturalidad basada en el reconocimiento de la pluralidad epistemológica. En León Olivé et al., Pluralismo epistemológico. Bolivia: ClACSO, pp. 19-30.

OREAlC/UnesCo (2017). Conocimiento Indígena y Politicas Educativas en América Latina. Santiago: UNESCO.

Pérez Luna, Enrique, Norys Alfonzo Moya y Antonio Curcu Colón (2013). Transdisciplinariedad y educación. Educere, 17 (56), 15-26.

Quintriqueo Millán, Segundo y Héctor Torres Cuevas (2012). Distancia entre el conocimiento mapuche y el conocimiento escolar en contexto mapuche. Revista Electrónica de Investigación Educativa, 14(1), 16-33.

Smith, Linda Tuhiwai (2002). Decolonizing Methodologies. Research and Indigenous Peoples. Auckland: Zed Books.

Spivak, Gayatri (1988). Can the subaltern speak? En C. Nelson and L. Grossberg (eds.), Marxism and the Interpretation of Culture. Basingstoke: Macmillan Education, pp. 271-313.

Urrieta, Luis Jr. (2013). Familia and Comunidad-Based Saberes: Learning in an Indigenous Heritage Community. Anthropology \& Education Quarterly, 44 (3), 320-335.

Universidad Veracruzana Intercultural (UVI) (s.f.) Criterios para la investigación vinculada para la gestión intercultural en la Universidad Veracruzana Intercultural. www.uv.mx/uvi/criterios-de-investigacion-vinculada-para-la-gestion/ 
